\title{
Téoros
}

Revue de recherche en tourisme

\section{Tourisme et aménagement : une relation amour-haine à Québec}

\section{Claude Dubé}

Volume 12, numéro 1, mars 1993

Québec, capitale et région touristiques

URI : https://id.erudit.org/iderudit/1078195ar

DOI : https://doi.org/10.7202/1078195ar

Aller au sommaire du numéro

Éditeur(s)

Université du Québec à Montréal

ISSN

0712-8657 (imprimé)

1923-2705 (numérique)

Découvrir la revue

Citer cet article

Dubé, C. (1993). Tourisme et aménagement : une relation amour-haine à Québec. Téoros, 12(1), 10-12. https://doi.org/10.7202/1078195ar d'utilisation que vous pouvez consulter en ligne.

https://apropos.erudit.org/fr/usagers/politique-dutilisation/ 


\title{
Tourisme et aménagement : une relation amour-haine à Québec
}

\author{
Claude Dubé*
}

Des 1975, un groupe de recherche de l'Université Laval( ${ }^{(1)}$ étudiait la perception du Vieux-Québec que pouvaient avoir les touristes americains, canadiens, montréalais et enfin québécois. En résumé, il se dégageait le consensus suivant: on vient dans le VieuxQuébec non pas pour voir les vieilles pierres mais plutôt pour voir des gens vivre dans de vieilles pierres.

Ceci confirmait lajustesse de la réorientation des operations de restauration du patrimoine architectural (dont Place Royale est le cas type à eviter) mais faisait surtout ressortir le rôle primordialdes résidents dans l'attraction d' un site aussi renommé que celui du VieuxQuébec. C'est cette relation que l'on souhaite en équilibre entre tourisme et aménagement, sorte de relation amour-haine, que nous aborderons spécifiquement pour le Vieux-Québec en tentant de faire ressortir son évolution au cours des ans.

Dans un rapport traitant des transports au centre-ville $e^{(2)}$, on citait quelques conclusions préliminaires de l'étude du groupe de l'Université Laval, notamment que les touristes rechercheraientd"abord un petit hôtelà cause de sa taille et de son aspect sympathique dans la trame urbaine du Vieux-Québec. Quelques mois avant la publication de ce rapport, une fuite permettait au journal Le Soleil un scoop bien orchestré qui arrêta le projet de construction d' une tour de 37 tétagesà côté de l'Hôtel Hilton, à l'endroit même où aujourd'hui on projette la construction d'un centre des congrès.

Cette même étude rapportait que plus de 80 \% de tous les groupes de touristes questionnés exprimaient le désir d'interdire ou de restreindre la circulation des voitures privées dans le Vieux-Québec. Nous verrons plus loin que les résidents sont toujours préoccupés de cette question, plus de 15 ans après cette constatation. Nous verrons aussi que le transport semble causer le plus de problèmes àune relation harmonieuse entre les résidents et les touristes.

Monsieur Claude Dubé, architecte et urbaniste, est directeur de l'Ecole d'archilecture de l'Université Laval.

\section{L'évolution du tourisme dans le Vieux-Québec}

Au fil des ans, l'ampleur des problèmes de circulation s'est atténuée à cause de deux grandsfacteurs: lepremierestledéplacement de certaines activités touristiques hors des murs du Vieux-Québec. Ainsi, les activités de soirées (bars, discothèques, restaurants) ont-elles migré vers les rues Grande-Allée. Cartier, etç; de même, les activités de magasinage se sont concentrées sur la rue Saint-Jean, hors les murs, et dans le quartier du PetitChamplain, en basse-ville. Cesdivers changements progressifs ont fait baisser la pression sur les besoins de déplacement dans l'espace restreint du Vieux-Québec intramuros.

Un deuxième facteur a agi progressivement, lui aussi: la réalisation de plusieurs améliorations physiques et réglementaires quant à la circulation et au stationnement (sens unique, vignettes, postes de taxi, etc.). On a réduit ainsi plusieurs causes de conflits.

En changeant ainsi, ce quartier a perdu des commerces de nature régionale (Holt Renfrew, Birks, etc.) pour offrir surtout des commerces utiles å la clientèle touristique au détriment, bien souvent, des besoins des résidents.

\section{L'évolution du tourisme}

Le tourisme, pour sa part, a évolué aussi pendant ce temps. Le point majeur est peutêtre l'accroissement très important du tourisme d'autocar qui devrait continuer a croître de façon significative dans les prochaines années. À la différence des touristes qui recherchent le petit hôtel, les longues promenadesetqui participentauxévénements culturels en salle ou sur la rue, le tourisme d'autocar est réglé selon un horaire précis. Au cours d'une journée typique, on passera de 2 a 3 heures dans le Vieux-Québec (une période aussi longue dans ce genre de visite témoigne de la grande importance touristique du site), pourensuite faire le tour de l' Iled'Orléans (en arrêtant 5 minutes à SaintePétronille); puis, on fera la Côte de Beaupré
jusqu'àSainte-Anneet, finalement, retour au point de départ pour le souper.

Les trajets sont bien réglés, avec des groupes dociles (généralement des gens d'un certain âge) où le temps en autocar est optimisé parce qu'il coûte moins cher parce que les risques (accident, oubli d"une personne, retards, etc.) sont minimes. Ceci permet de prévoir et de faire des ententes avantageuses avec les hôtels et restaurants pour toute la durée de la période touristique. (Le pouvoir de négociation de ces firmes de service est énorme: environ 20 millions de dollars de chiffre d'affaires annuellement). On consomme ainsi du paysage derrière des vitres teintées dans un environnement climatisé.

Cette évolution a déjà engendré des modifications importantes, comme l'achat de petits hôtels pour les groupes de noliseurs et évidemment, laprésence constante des autocars dans les rues à faible gabarit du VieuxQuébec. La possibilité que tout véhicule, autocar compris, puisse faire maintenant du sightseeing dans le Vieux-Québec accroît la présence de gros véhicules. Il y a une dizaine d'années, la CTCUQ avait le monopole des visites guidées dans la région. La perte de ce monopole de la CTCUQ s'inscrit dans le courant de la déréglementation et ce droit pour tous les autocars de faire du sightseeing serait même protégé par la Chante des droits et libertés. Mais les municipalités se retrouvent prises ainsi en otage par cette activité touristique.

\section{Le réveil des résidents}

Le déplacement de diverses fonctions hors les murs de la vieille ville et la concentration de commerces et de services répondant aux besoins touristiques et gouvernementaux, de préférenceàceux des résidents, ont provoqué une reprise en main du quartier par ceux-ci vers 1990.

En se fondant sur des analyses intéressantes etélaborces, le Comitédescitoyens du VieuxQuébec a adopté plusieurs documents de prises de positions touchant tous les aspects de la vie de quartier, sans négliger ou laisser 
pour compte la vocation hautement touristique de ce secteur.

C'est toujours à propos de la circulation et du stationnement que les contraintes dues au tourisme ressortent. Ainsi, dans un mémoire à l'adresse de la Ville de Québec, le 15 mai 1990 , le comité propose neuf interventions ${ }^{(3)}$ pour sune réappropriation de la rue dans le Vieux-Québec au bénéfice premier de la vie du site lui-même et au bénéfice de tous ses résidents, commerçants, travaillants et visiteurs: la rue pour la vie du quartier, la rue pour touss ${ }^{(4)}$.

Plusieurs de ces propositions touchent au tourisme mais $l^{*}$ une d'elles est spécifique à ce sujet:

\section{Contenir la circulation \\ des autobus de tourisme.}

Laisser les autobus de croisière et les gros autobus de tour de ville dévaler les rues étroites, obstruer la vue par leurs silhouettes agglutinées et massives en stationnement, infester l'air des bruits et des gaz de leurs moteurs qui tournent indéfiniment à l'arrêt, c'est compromettre gravement le caractère et l'atmosphèredu Vieux-Québec. Des règles doivent s'appliquer à l'arrêt et au stationnement des autobus de croisière qui amènentdes touristes. Uneorganisation différente des tours de ville doit retenir aux portes les gros autobus en guidant les visites soit à pied, soit en minibus,.s.

Pourquoi cette vision si négative alors que des quelque 200 autobus franchissantla Porte Saint-Louis en une journée en été, environ la moitié sont des autobus de la CTCUQ? Je dois porter mon chapeau de résident (plusieurs années) et de travailleur dans le VieuxQuébec pour tenter une interprétation. Ma perception est la suivante: je ne perçois que faiblement les inconvénients des autobus de la CTCUQ parce qu'ils sont toujours en mouvement et qu'ils offrent un service qui m'estaccessible. Ma perceptiondes autocars nolisés est cependant différente, car c'est l'autocar du mardi et du jeudi midi qui est stationné (très souvent son moteur et sa climatisation en marche) devant le même restaurant, du début mai à la fin septembre. Si encore ce n'était qu'un autocar. Mais quand il y en a 7 ou 8 en permanence (bien qu'ils se remplacent constamment) sur laplace devant le Château Frontenac, je me sens envahi et agressé.

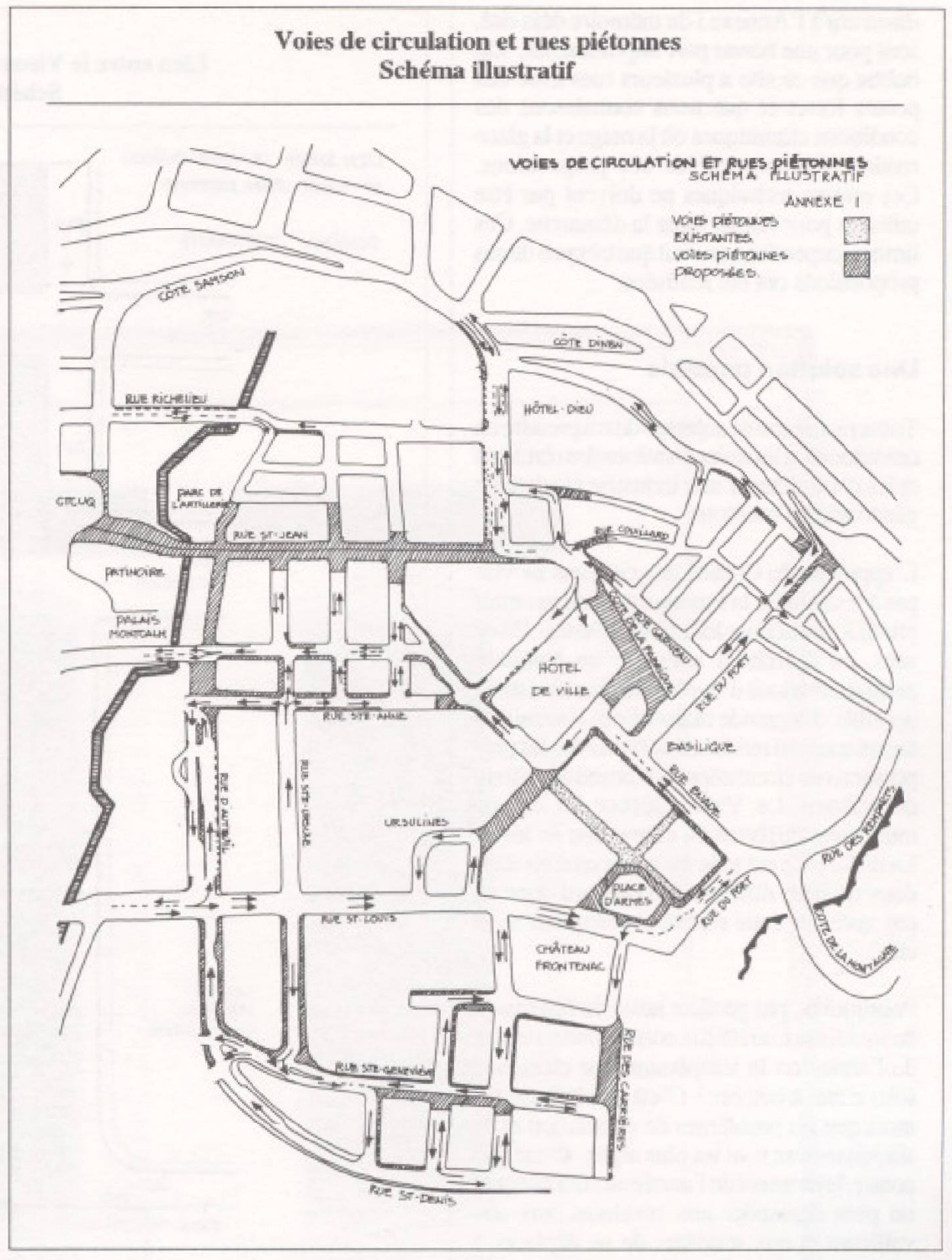

La perception qu'en ont les résidents de l'extérieur du Vieux-Québec sera différente, probablement moins acerbe. La question est de savoir si la possibilite de vivre quotidiennement dans un site si magnifique compense pour les inconvénients. Il semble que le Comité des citoyens ait répondu non a cettequestiondansleurspropositions (schéma illustratif), sorte de plan global de circulation pour le Vieux-Québec.

La vision du Comité des citoyens cherche évidemment à transformer le Vieux-Québec en un lieu agréable à vivre. En ce sens, la priorité aux piétons et les restrictions aux véhicules motorisés vont aussi dans le sens de la perception des touristes lors de l'en= quête de 1975. En effet, si ce quartier devient plus agréable à vivre, sa population aura tendance à augmenter et les touristes verront mieux la vie des résidents dans un quartier ancien.

Cependant, le tourisme d' autocar necherche pas a voir des gens vivre dans de vieilles pierres. Ce tourisme est consommateur de monuments, de paysages, de spectaculaire et la vitesseàlaquelle il est contraint d' absorber cette information l'empêche fort probablement de porter la moindre attention aux gens qui peuplent ces lieux.

Toutefois, au-delà des principes, les propositions du Comité des citoyens, selon le schéma 
illustratif à l'Annexe I du mémoire déjà cité, sont pour une bonne part impraticables. On oublie que ce site a plusieurs nes avec des pentes fortes et que nous connaissons des conditions climatiques où la neige et la glace rendraient impraticables ces propositions. Ces erreurs techniques ne doivent pas être utiliś́es pour rejeter toute la démarche. Ces limites, cependant, ont fait que très peu deces propositions ont été réalisées.

\section{Une solution possible}

Toute recherche de solution devra prendreen considération les revendications des résidents et les contraintes d'une industrie touristique génératrice d'emplois.

L'approche du Comité des citoyens ne vise pas à restreindre la fonction touristique mais plutôtta à en atténuer les effets néfastes. En ce sens, on cherche à atteindre un équilibre permettant à tous d'évoluer le plus librement possible. La grande majorité des contraintes techniques qui rendent impraticables les propositions de circulation du Comité découlent du climat. Le Vieux-Québec en été est totalement différent du même lieu en hiver. La différence est telle qu'on se croirait dans deux mondes différents. Pourquoi donc ne pas accepter cette réalité et composer avec elle?

Pourquoi ne pas profiter aussi du fait que la haute saison tou ristique corres pond au temps de l'année où la température est clémente, soit de mai à octobre? C'est pendant ces six mois que les problèmes de circulation et de stationnement sont les plus aigus. C'est, par contre, le moment de l' année au cours duquel on peut demander aux résidents, aux travailleurs et aux touristes de se déplacer à pied, la plupart du temps, dans le vieux quartier.

Le plan présenté par le Comité des citoyens, avec certains ajustements techniques, pourrait servir de base à l'élaboration d'un plan acceptable par la Ville de Québec pour ce secteur durant la saison d'été.

Un autre plan, plus permissif, pourrait être développé pour la période plus froide alors que les conflits entre les touristes et les résidents sont presqu'inexistants; on réduirait alors le nombre et l'importance des trajets à pied obligatoires pour tous les groupes d'usagers de la vieille ville.

\section{Lien entre le Vieux-Québec et Saint-Baptiste Schéma illustratif}

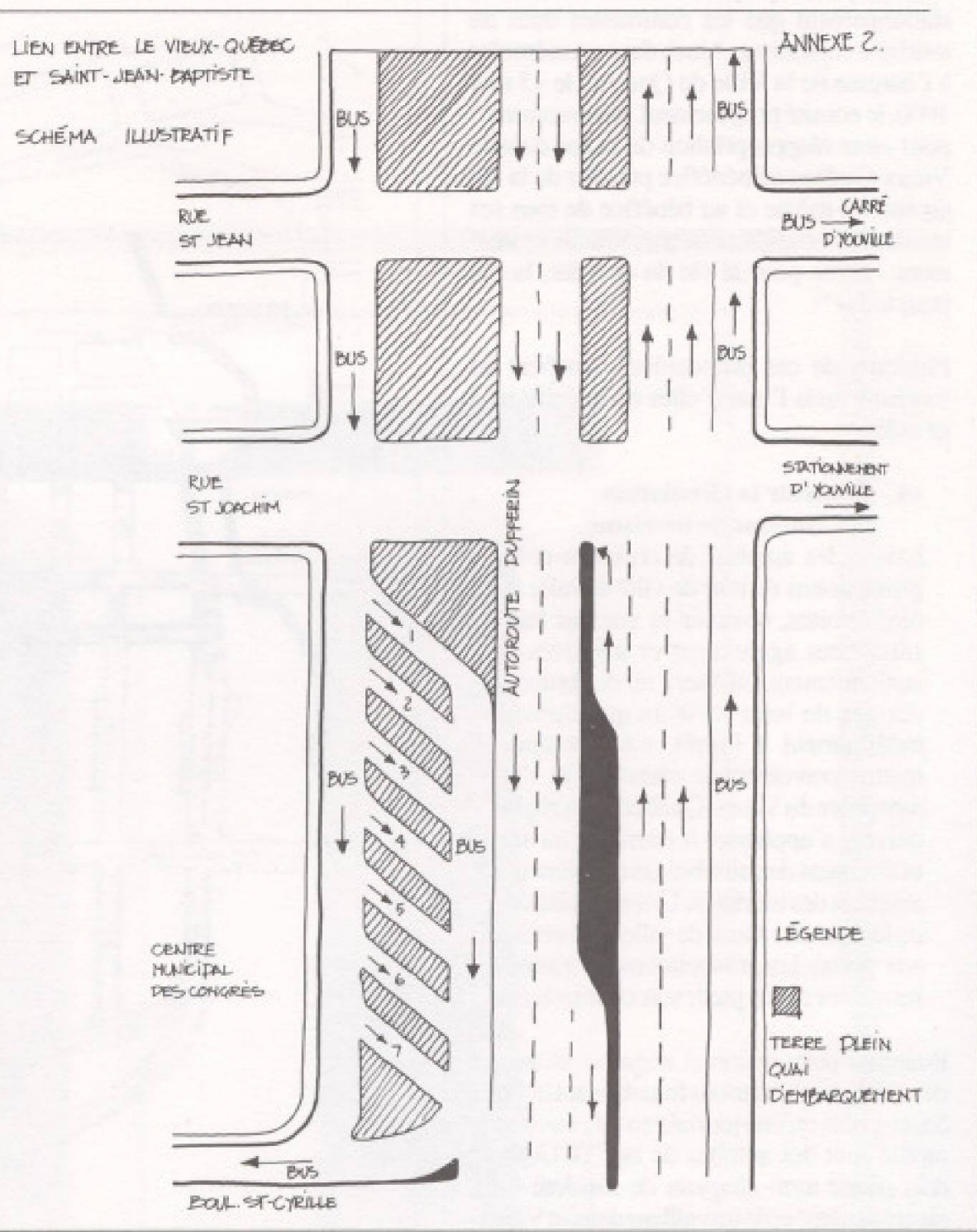

\section{Est-ce réaliste?}

Les avantages que pourraient en tirer tous les utilisateurs méritent qu'on étudie cette proposition.Ceci ne réglerait sûrement pas ainsi tous les problèmes du Vieux-Québec mais on atténuerait pour le moins les impacts négatifs de la fonction touristique. En ces temps de récession, encourager une industrie florissante reste suirement souhaitable si, en même temps, on améliore la qualité de vie des résidents.

\section{RÉFÉRENCES}

(1) Projet de recherche du Laboratoire de gégraphie culturelle del'Université Laval, sous la direction de monsieur Marcel Bellanger, 1975.

(2) Commissiond'aménagement de la Communauté urbaine de Québec, Les transports au centreville de Oubbec, mai 1976. $200 \mathrm{p}$.

(3) Liste des 9 interventions: 1-Donner la priorite aux personnes: 2-Detoumerlacirculation de transit; 3 Dissuader de la balade en voiture; 4- Contenir la circulation des autobus de tourisme; 5 - Miser sur le transport en commun; 6-Canaliser la circulation principale; 7-Limiter la circulation dans certaines zones; 8-Créer des espaces piétonniers; 9 - Relier le Vieux-Québec au quartier Saint-Jean Baptiste.

(4) La rue pour toue et la vie du Ouartier, mémoire du Comité des citoyens du Vieux-Québec, présenté di la Vile de Quebec le 15 mai 1990. 